\title{
A Two-stage Coordinated Strategy for Integrated Exploration of Multi-robot Systems
}

\author{
Xue-Feng DAI ${ }^{a,{ }^{*}}$, Lai-Hao JIANG ${ }^{b}$
}

School of Computer and Control Engineering, Qiqihar University, Heilongjiang, 161006, China

adaixuefeng203@163.com, 'lhjiangkyo@126.com

"Corresponding author

Keywords: Multi-robot Systems, Exploration, Coordination, Entropy, Auction Algorithm.

\begin{abstract}
To improve performance for integrated exploration algorithms of multi-robot systems, a two-stage coordinated strategy is proposed in this paper. In the first stage, all robots explore unknown environments by a kind of forth and back trajectories which are shaped by social entropy and a voting mechanism. In the second stage, the solving process of coordination problem for multi-robot systems is modeled as a blackboard system, and each robot contributes a solution to the question determined by a capacity constrained auction algorithm. The proposed approach is verified by computer simulations for obstacle free environments.
\end{abstract}

\section{Introduction}

Integrated exploration approaches which considered coordination, mapping, localization and motion control tasks simultaneously is preferred. However, only a few of results about the topic have been reported [1-2]. On the other hand, a great deal of results has been proposed for the coordination of multi-robot systems. There is market economy based approaches [3-6], evolutionary computing based approaches [7-9], decision-theoretic approach [10], etc. These methods realized task assignments. Meanwhile, they considered route planning under intra-path constraints [7], cooperation among robots [3], and dispersion of robots over the environment [10] in the explorations of unknown environments.

Shannon's information entropy has been utilized as a measure for uncertainty about robots [8]. Hierarchic social entropy that is an application of Shannon's information entropy was introduced as a continuous and quantitative measure for robot group diversity [11]. In this paper, the property that social entropy can represent spatial distribution of homogeneous robots is utilized to shape trajectories of robots over an unknown environment. Moreover, the exploring trajectories of all robots are used to partition operational environment into a set of sections. Notwithstanding the partitioning is not balanced, the subsequent auction actions will balance tasks among robots.

Recently, a three-level optimization algorithm was proposed to coordinate emergency medical service robot team [12]. In the approach, a globally efficient solution at each level is obtained by a medication of auction algorithm. The time complexities of sing-item, multi-item (greedy), multi-item (optimal), and combinational auctions were compared in [6]. Moreover, computational complexity exists in determining the cost to perform a task that requires solving path planning of multi-robot systems; an instance is traveling salesman problem (TSP) which is NP-hard. Differently with the available approaches, the auction algorithm is utilized to solve a blackboard system which represents the coordination problem in the proposed approach. At the same time, the dispersion of robots over the environment is considered.

Zigzag motion is an effectively way to cover the environment the robots located [13]. Inspired by the result meanwhile considering error corrections, a generalized forth and back -- rectangle trajectories are adopted by all robots of a multi-robot system in the present work. The rest of this paper is organized as follows. Section 2 discusses coordination based on social entropy. Section 3 discusses 
coordination formulation and solving by an auction algorithm. Section 4 validates the proposed approach by computer simulations. And Section 5 concludes the paper.

\section{Entropy Based Exploration}

As shown in Fig.1, there are two robots $R_{1}$ and $R_{2}$, and the two black circles are the initial locations of the two robots, respectively. All the four longer edges in the two trajectory rectangles are vertical. For convenience of discussion, it is named that both $R_{1}$ and $R_{2}$ has vertical auxiliary exploring direction. Similarly, there is horizontal auxiliary exploring direction. The direction that a robot will follow when it has closed a rectangle trajectory for the first times is named as auxiliary exploring direction.

It is assumed that all robots have no knowledge about the environment except relative poses between robots [2]. In order to simplify the discussion, the moving directions for each robot is restricted to up, down, left and right.

At the beginning of exploration, the social entropy is calculated based on the relative distances by each robot. Then, each robot selects a candidate exploring direction by minimizing the entropy. As discussed in [13] and shown in Fig.1, only in the case that all robots have parallel auxiliary exploring direction can they cooperatively explore a region of the environment well. So, a common auxiliary exploring direction should be determined first.

To obtain the common auxiliary exploring directions, a voting mechanism based on all candidate exploring directions is utilized, i.e., if the sum of numbers of up and down candidate exploring directions is bigger than the sum of numbers of left and right candidate exploring directions, then the common auxiliary moving direction is vertical (consists of up and down directions); else the common auxiliary exploring direction is horizontal (consists of left and right directions).

If a robot has candidate exploring direction consistent with the common auxiliary exploring direction, then no re-selection of auxiliary exploring direction is needed; else a re-selection of an auxiliary exploring direction from two consistent directions is needed. The re-selection is based on minimizing the social entropy. After a robot reached at border along its auxiliary exploring direction, its temporary exploring direction is determined by minimizing the entropy too.

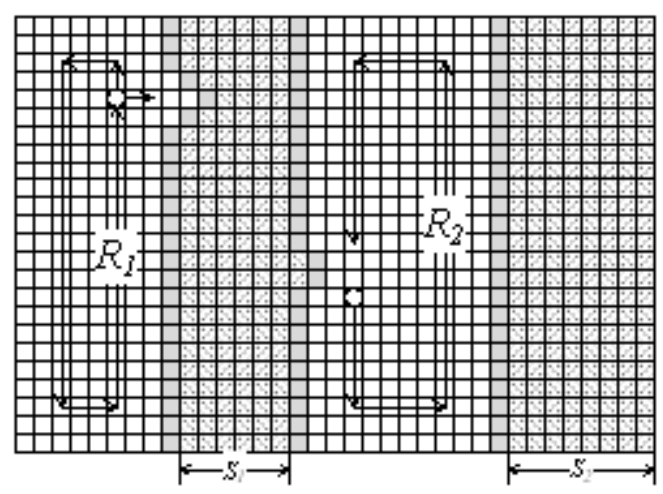

Fig.1. Demonstration for cooperative exploring trajectories and sections

Besides above auxiliary and temporary directions, subsequent exploring directions are determined to form a rectangle. The exploration discourse of a robot that started from its initial location is named as the first exploration stage. Whenever a robot cannot explore unknown area in the first stage, it is said that the robot switched to the second stage. A flowchart describing the proposed coordinated strategy for a robot is given in Fig.2. 


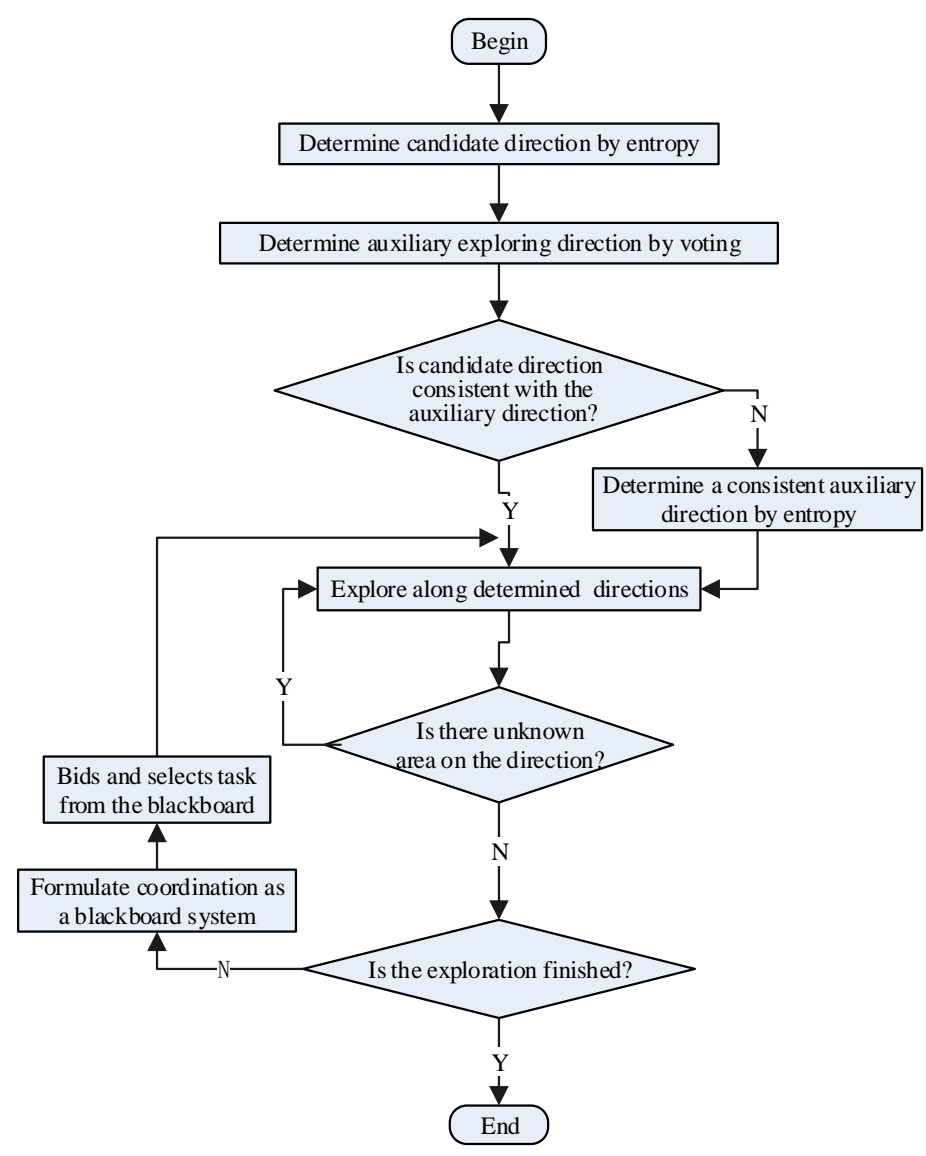

Fig.2 A flowchart of two-stage coordinated strategy

\section{Auction Based Exploration}

At the beginning of the second stage, there are at most $n$ sections partitioned by the explored areas and borders of the environment. For example, there are two sections $S_{1}$ and $S_{2}$ on the environment shown in Fig.1. In order to assign sections to robots, and disperse all robots over the environment, each section is attributed by $\left\langle d_{j}, b_{j}, n_{c j}, q_{j}\right\rangle(\mathrm{j}=1, \ldots, \mathrm{n})$, where $d_{j}$ is the width, $b_{j}=\left\langle l_{j}, u_{j}\right\rangle$ the border coordinates, where $l_{j}$ and $u_{j}$ are the lower and upper coordinates, respectively, $n_{c j}$ is the capacity, and $q_{j}=\{$ unscanned, to_be_scanned, being_scanned $\}$ is a status of the section. $n_{c j}$ means the maximal number of robots that a section $S_{j}$ with status unscanned can contain. It is calculated according to the size of the section by the following algorithm.

Algorithm 1. Section capacity calculation.

1) If $d_{j}>0$, then $n_{c j}=1$.

2) Construct a temporary variable set $D_{t}=\left\{d_{t j} \mid j=1, \ldots, n\right\}$, initially $d_{t j}=d_{j}(j=1, \ldots, n)$.

3) If $\sum_{j=1}^{n} n_{c j}<n_{a}$, where $n_{c j}$ is the current capacity value, $n_{a}$ is the number of robots taking part in the task assignment, then go on; else end.

4) $p=\arg \max _{j=1, \ldots, n} D_{t}, n_{c p}=n_{c p}+1$, and $d_{t p}=d_{t p}-d_{0}$, where $d_{0}$ is the maximal area a robot can scan in a task assignment period.

5) If $\neg \exists d_{t j}>0$, then end; else go to step 3).

It must be marked that $d_{0}$ in 3 ) is represented in form of section width, and $d_{0}=2 r$ in ideal case, where $r$ is the detecting rang of the robot considered. The coordination of the multi-robot system is to force all widths of explored sections to zeros. Then, the coordination is formulated as a blackboard system 


$$
\sum_{j=1}^{n} d_{j}(k) \rightarrow 0
$$

where $d_{j}(k) \geqslant 0$ is current width of the $j$ th unknown section, $k(k=1,2, \ldots)$ is a series of discrete time instants when any robot completed its task, and $n$ is the size of the multi-robot system considered. All robots share the unexplored information and strive to scan the remained portion of the environment. So, a sequential single-item auction algorithm is utilized to solve (1). In the auction algorithm, utility of each section is selected as the width of each unknown section, and the cost is taken as the distance from the robot current location to the nearest border of the section. Afterwards, the section is divided into at most $n_{c j}$ sub-sections (hereafter section) and the status of each section is set to to_be_scanned, and is changed to being_scanned after the robot reached at the section. The auction actions are asynchronous. The auctioneer assign total at most $n_{c j}$ robots to section $j$ by minimizing the individual evaluations (utility minimize cost).

If the sum of total available capacity is less than the number of free robots, the remained robots bid for sections with the status variables being to_be_scanned. If there are still free robots after above assignment, the robots bid for sections with status variable being being_scanned. After a robot has closed its trajectory, then $d_{j}(k)=\mathrm{d}_{\mathrm{j}}(k-1)-\Delta d_{j}(k)$, where $\Delta d_{j}(k)$ is the area actually covered by the robot from time $k-1$ to $k$. As soon as a robot closed its trajectory, the capacities of all available sections are re-calculated.

\section{Computer Simulations}

Computer simulations have been conducted through Matlab ${ }^{\mathrm{TM}} 7.1$ on a personnel computer with Intel ${ }^{\circledR}$ Core(TM) i5-3470 CPU and 4GB RAM. An obstacle free indoor environment which has area of $100 \times 100 \mathrm{~m}^{2}$ is selected as simulated scenario. The proposed two-stage coordinated approach (TSCA) is compared with decision-theoretic approach (DTA) [10], and repeated auction approach (RAA) [4].

All robots have the same moving speed of $0.20 \mathrm{~m} / \mathrm{s}$, sensing range $r=4 \mathrm{~m}$, and the waiting time at an auction period is $5 \mathrm{~s} .30$ runs for each algorithm with the individual team sizes have been conducted. At the beginning of each run, all robots are randomly located on the environment. The purpose of the exploration for the multi-robot system is completely covering the environment. Fig.3 (a) and (b) shows that the proposed approach saved exploration time, and coordinate framework transformation times, respectively. The reduction of coordinate transformation times save computational cost of postprocessing to obtain a global map [14]. Fig.3 (c) and (d) shows that our approach simplified task assignment procedure. 


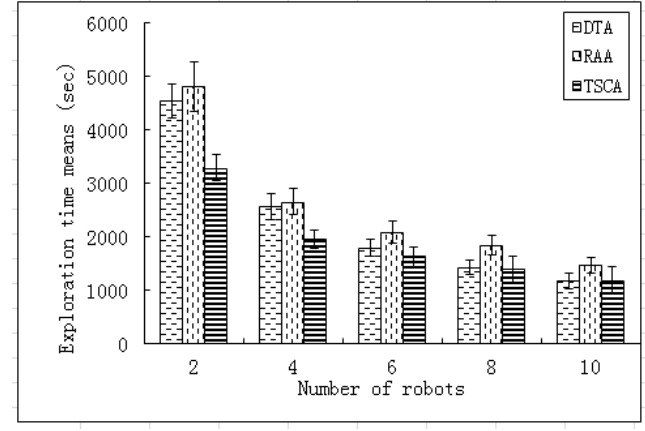

(a) Exploration times

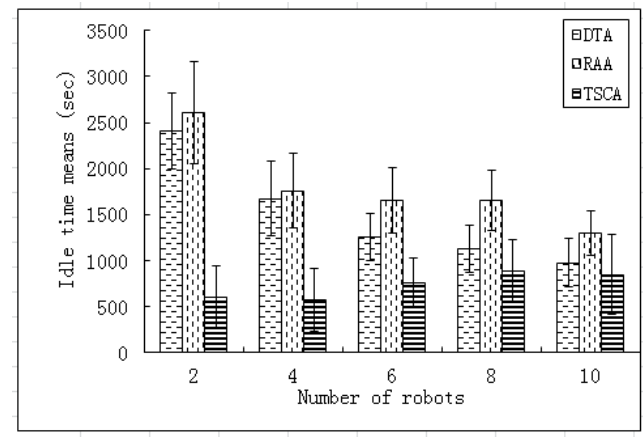

(c) Idle times

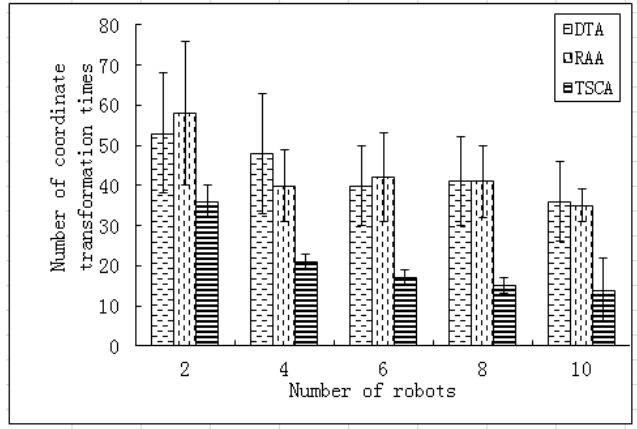

(b) Coordinate transformation times

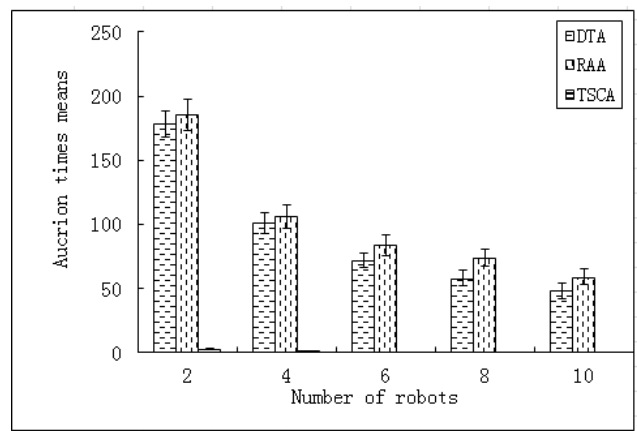

(d) Auction times

Fig.3. Performance comparison for the three approaches

Fig. 4 shows trajectories for a case of two robots coordinated by the three algorithms. It is shown that the trajectories of robots coordinated by the proposed approach are more regular and smoother than those of the other two coordinated algorithms.

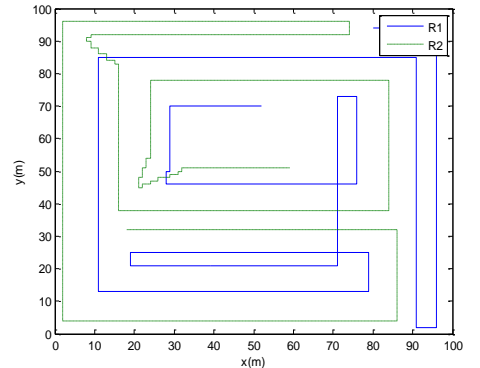

(a)Trajectories for DTA

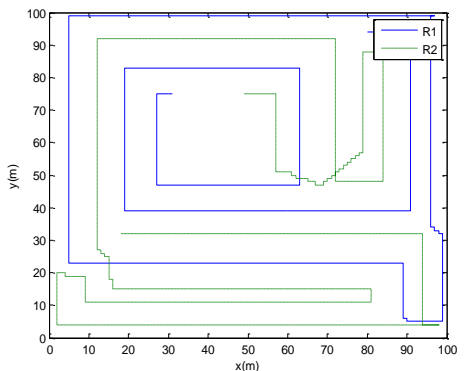

(b)Trajectories for RAA

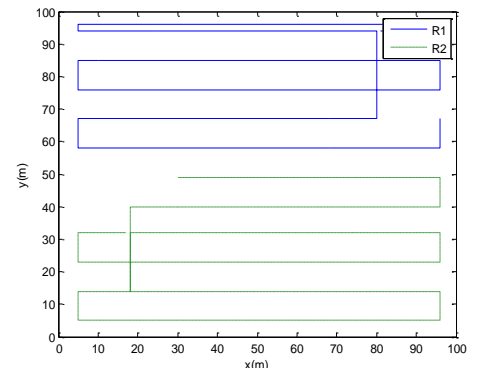

(c) Trajectories for TSCA

Fig. 4. A case for trajectory comparisons

\section{Conclusions}

A simple yet effective coordination strategy has been proposed for integrated exploration of unknown obstacle free environments of multi-robot systems. How to endow the proposed approach an ability to deal with coordination in obstacle occupied environments is future work.

\section{Acknowledgement}

This research was financially supported by the National Science Foundation of China under Grant 61672304, and the Natural Science Foundation of Heilongjiang Province, China, under Grant F201331. 


\section{References}

[1] M. Julia, O. Reinoso, A. Gil, M. Ballesta, L. Paya, A hybrid solution to the multi-robot integrated exploration problem, Eng. Appl. Artif. Intel. 23 (2012) 473-486.

[2] M. Julia, A. Gil, O. Reinoso, A comparison of path planning strategies for autonomous exploration and mapping of unknown environments, Auton. Robots 33 (2012) 427-444.

[3] T. Andre, C. Bettstetter, Collaboration in multi-robot exploration: To Meet or not to Meet? J. Intell. Robot. Syst. 82 (2016) 325-337.

[4] M. Nanjanath, M. Gini, Repeated auctions for robust task execution by a robot team, Robot. Auton. Syst., 58 (2010) 900-909.

[5] W. Sheng, Q. Yang, J. Tan, N. Xi, Distributed multi-robot coordination in area exploration, Robot. Auton. Syst. 54 (2006) 945-955.

[6] M.B. Dias, R. Zlot, N. Kalra, A. Stentz, Market-based multirobot coordination: a survey and analysis, Proc. IEEE 94 (2006) 1257-1270.

[7] E.G. Jones, M.B. Dias, A. Stentz, Time-extended multi-robot coordination for domains with intra-path constraints, Auton. Robot. 30 (2011) 41-56.

[8] Q.-C. Huang, and R.-H. Luo, An active cooperative localization method for mobile robots, Acta Electronica Sinica 38 (2010) 2644-2648 (in Chinese).

[9] K. Jose, D.K. Pratihar, Task allocation and collision-free path planning of centralized multi-robots system for industrial plant inspection using heuristic methods, Robot. Auton. Syst. 80 (2016) 34-42.

[10] W. Burgard, M. Moors, C. Stachniss, F.E. Schneider, Coordinated multi-robot exploration, IEEE Trans. Robot. 21 (2005) 376- 86.

[11] T. Balch, Hierarchic social entropy: An information theoretic measure of robot group diversity, Auton. Robot. 8 (2000) 209-237.

[12] M. Lujak, H. Billhardt, S. Ossowski, Distributed coordination of emergency medical service for angioplasty patients, Ann. Math. Artif. Intell. 78 (2016) 73-100.

[13] S.X. Yang, C. Luo, A neural network approach to complete coverage path planning, IEEE Trans. Syst. Man Cybern.-Part B: Cybern. 34 (2004) 718-725.

[14] C. Estrada, J. Neira, J.D. Tardos, Hierarchical SLAM: real-time accurate mapping of large environments, IEEE Trans. Robot. 21 (2005) 588-596. 